\title{
Erratum: Hydrodynamics and Time Correlation Functions for Cellular Automata
}

\author{
M. H. Ernst ${ }^{2}$ and J. W. Dufty ${ }^{3}$
}

Received April 17, 1990

The Green-Kubo formulas (5.12) for the diffusivities of the spurious diffusive modes in CA fluids are correct, but the expressions for the associated scalar transport coefficients $\kappa_{1}(s)$ and $\kappa_{2}(s)$ in (5.13) are not. They should read

$$
\begin{array}{r}
\kappa_{1}(s)=\left(\chi_{l}\right)^{-1} \sum_{t=0}^{*} e^{-s t} \sum_{\mathbf{r}}(-1)^{t+\boldsymbol{\beta} \cdot \mathbf{r}}\left\langle\sigma_{\perp}(0,0) \sigma_{\perp}(\mathbf{r}, t)\right\rangle \\
\kappa_{1}(s)+\kappa_{2}(s)=\left(\chi_{l}\right)^{-1} \sum_{t=0}^{*} \mid e^{-s t} \sum_{\mathbf{r}}(-1)^{t+\beta \cdot \mathbf{r}}\left\langle\sigma_{\|}(0,0) \sigma_{\|}(\mathbf{r}, t)\right\rangle
\end{array}
$$

where $\sigma_{\perp}$ and $\sigma_{\|}$are components of the stress tensor orthogonal and parallel to any of the vectors $\hat{\beta} ; \sigma_{\| \mid}=\hat{k}_{i} \hat{\beta}_{j} \sigma_{i j}$ and $\sigma_{\perp \perp}=\hat{k}_{i} \hat{\beta}_{\perp_{j}} \sigma_{i j}$. Here $\hat{\beta}_{\perp}$ is orthogonal to $\hat{\beta}$ and $\sigma_{i j}$ is the stress tensor. These results follow from the fact that the staggered diffusion coefficient $A_{\beta}(\hat{k}, s)=\hat{k}_{i} \hat{k}_{j} \Delta_{i j}(\boldsymbol{\beta})$ is related to a second order tensor field [see Eq. (5.11)] with the representation $\Delta_{i j}(\boldsymbol{\beta})=\kappa_{1}(s) \delta_{i j}+\kappa_{2}(s) \hat{\beta}_{i} \hat{\beta}_{j}$.

\footnotetext{
${ }^{1}$ This paper originally appeared in J. Stat. Phys. 58:57 (1990).

${ }^{2}$ Institute for Theoretical Physics, 3508 TA Utrecht, The Netherlands.

${ }^{3}$ Department of Physics, University of Florida, Gainvesville, Florida 32611.
} 